\title{
Le radar en bande $X$ : un outil au service de l'étude des événements précipitants
}

\author{
Yvon Lemaître ${ }^{(1)}$, Jean-François Rysman ${ }^{(1)}$, Sébastien Verrier ${ }^{(1)}$, \\ Emmanuel Moreau ${ }^{(2)}$ \\ (1) Laboratoire atmosphères, milieux, observations spatiales (Latmos), CNRS, \\ Université de Versailles Saint-Quentin-en-Yvelines (UVSQ), \\ 11 boulevard d'Alembert, 78280 Guyancourt, France \\ (2) Novimet, 11 boulevard d'Alembert, 78280 Guyancourt, France
}

\section{Résumé}

Ce papier illustre le potentiel d'application de la mesure de pluie par radar polarimétrique et Doppler en bande $X$. Il considère la mesure dans les régions à relief particulièrement accidenté et se focalise sur les propriétés statistiques et les variabilités spatiales et temporelles de la pluie. Il s'appuie sur les premières mesures de ce type réalisées depuis début 2009 de façon continue dans la région AlpesMéditerranée par le radar Hydrix ${ }^{\circledR}$.

\section{Abstract}

\section{X-band radar: a tool for examining rainfall events}

This paper illustrates the potential of the application of rain measurements made with polarized $X$-band and Doppler radar. It considers these measurements in area with particularly broken relief and focuses on the statistical properties and on spatial and temporal variability of rain. It is based on the first measurements of this type carried out continuously by the Hydrix radar in the "Alps-Méditerranée" Region since the beginning of 2009.

\section{Introduction}

Ces dix dernières années ont vu apparâ̂tre un besoin grandissant d'une meilleure connaissance de la variabilité des précipitations et de leurs propriétés statistiques. Ce besoin s'est fait sentir dans plusieurs domaines de recherche, qui vont de la mesure de la précipitation à la réponse des précipitations aux changements climatiques.

On peut ainsi citer les besoins d'identification des processus gouvernant les événements extrêmes, d'amélioration et de validation statistique des modèles météorologiques et par voie de conséquence d'amélioration de la prévision des événements extrêmes. Dans le domaine de l'hydrologie, on peut également rappeler l'importance de la variabilité spatiale de la pluie sur la modélisation hydrologique pluie-débit.

On peut également citer le besoin croissant de disposer de méthodes de « descente d'échelle » (désagrégation ou downscaling) pour des études d'impact ou pour inférer la variabilité à fine échelle à partir de mesures de résolution plus grossière réalisées par exemple par les moyens satellitaires ou les modèles de circulation générale (Michelangeli et al., 2009 ; Vrac et al., 2012).

Dans le domaine de la mesure de pluie qui se réalise à l'aide de moyens très diversifiés, in situ, par télédétection active ou passive au sol, ou à partir de l'espace, se pose la question de la représentativité de ces mesures réalisées avec des résolutions spatiales et temporelles très différentes et la nécessité de pouvoir les comparer ou les étalonner entre elles.

D'un point de vue plus pratique pour les études de processus, l'analyse statistique des précipitations permet d'identifier à la fois les caractéristiques moyennes et extrêmes d'une région géographique donnée. Ceci permet ainsi d'orienter les études de cas, nécessitant des analyses lourdes et complexes, sur des situations typiques ou des situations posant clairement un problème de prévision.

Ces besoins sont particulièrement importants dans le contexte de la prévision climatique régionale des événements précipitants. Ainsi, pour le bassin méditerranéen, des modifications importantes du cycle de l'eau sont attendues (Drobinski et al., 2013 ; Ducrocq et al., 2013), avec en particulier moins de précipitations mais des événements plus intenses (IPCC, 2007). Cependant, ces prévisions sont encore entachées d'erreurs importantes imputables à des effets de petites échelles et très localisés tels que les effets orographiques, qui modulent fortement les forçages dynamiques et thermodynamiques des précipitations. Cette difficulté, identifiée lors des événements catastrophiques rencontrés dans le passé (voir par exemple le cas de Vaison-la-Romaine en septembre 1992), nécessite de réaliser des études très locales et à fine échelle.

Parallèlement à cet engouement pour les propriétés statistiques de la pluie, la dernière décennie a vu l'avènement de 
moyens adaptés à ce type d'étude tels que le radar Doppler polarimétrique. Celui-ci permet en effet d'effectuer une caractérisation de la pluie avec des résolutions spatiales et temporelles fines et sur de longues durées grâce aux progrès réalisés sur la fiabilisation des composants radar. Cependant, la mise en œuvre de tels radars dans ces régions accidentées est rendue difficile par le relief, qui peut affecter la mesure radar par effet d'écran ou de masque partiel ou sous forme d'écho de sol.

Dans ce contexte, le radar Doppler et polarimétrique Hydrix ${ }^{\circledR}$ a été installé en 2007 dans une des régions où les précipitations intenses sont fortement influencées par l'orographie. Ce nouveau radar fonctionnant en bande $\mathbf{X}$ présentait l'avantage, par rapport aux radars pluie plus conventionnels (en bande $\mathbf{S}$ et $\mathbf{C}$ ) exploités jusqu'à maintenant, d'être de taille beaucoup plus petite, permettant ainsi son installation sur des infrastructures légères et surélevées tel que tours de télécom, châteaux d'eau, silo à grains ou toit d'immeuble. Cette plus faible longueur d'onde $(3,2 \mathrm{~cm})$ a une incidence directe sur le diamètre d'antenne, passant de 4-6 mètres pour des radars en bande C-S à $\sim 1,5 \mathrm{~m}$ pour les radars en bande X. Par ailleurs, les radars en bande $\mathrm{X}$ sont moins sensibles aux échos parasites tels que ceux issus du sol ou de la mer. Cependant, ce type de radar n'était pas exploité jusqu'à récemment, car dans cette bande de fréquence le signal est fortement atténué en présence de fortes précipitations.

L'avènement de techniques originales de correction du signal radar de cette atténuation, exploitant en particulier la double polarisation, a permis très récemment de surmonter cette difficulté (Testud et al., 2000 ; Moreau et al., 2009 ; Diss et al., 2009). Cette capacité polarimétrique permet de plus d'améliorer l'élimination des échos non météorologiques et de fournir des informations complémentaires telles que le type d'hydrométéore, information exploitable et utile dans les algorithmes d'estimation de la pluie à partir de la mesure radar. Pour finir, l'information Doppler peut être traitée pour reconstituer le vent dans le volume d'échantillonnage à l'aide de différentes méthodes d'inversion développées dans le passé pour des applications de recherche (Scialom et Lemaitre, 1990 ; Chong et Testud, 1996 ; Beck et Bousquet, 2013).

Dans la suite, après une brève description du radar, des données exploitées et de la zone d'étude, on illustrera le poten- tiel d'un tel outil pour analyser finement les propriétés statistiques et les variabilités spatiales et temporelles de la pluie. Enfin, un exemple de produits obtenus pour deux cas observés pendant la première campagne d'observation spéciale (SOP1) du projet HyMEX est présenté.

\section{Le radar Hydrix, bande X, Doppler et polarimétrique du mont Vial}

Le radar Hydrix, propriété du CNRS, a été conçu par la société Novimet dans le cadre d'une convention CNRS-Novimet, pour des applications hydrologiques opérationnelles. Il opère de façon continue depuis 2008 dans le sud de la France sur le mont Vial $(1500 \mathrm{~m})$ à $25 \mathrm{~km}$ au nord de Nice dans les Alpes-Maritimes. En complément des balayages d'antenne à basses élévations destinées aux applications hydrologiques, il réalise à la demande des balayages à hautes élévations pour les activités de recherche du Latmos.

Les caractéristiques techniques de ce radar sont les suivantes : il présente une ouverture de faisceau de 1,5 degré obtenue avec une antenne de 1,5 $\mathrm{m}$ de diamètre. Son antenne est de type offset (à foyer ou source décalés), produisant dans son diagramme de rayonnement des lobes latéraux (ou secondaires) particulièrement faibles $(<-30 \mathrm{~dB})$, ce qui améliore la visibilité radar en terrain complexe en minimisant les échos de sol vus par ces lobes.

Ce radar fonctionne sans radôme, ce qui permet d'éviter le sévère affaiblissement du signal lié à la pellicule d'eau qui se forme sur celui-ci lorsqu'il pleut (Frasier et al., 2013) et donc de prévenir l'apparition d'un biais variable sur l'étalonnage du radar en présence de pluie. L'autre avantage est la masse totale relativement faible du système (environ $350 \mathrm{~kg}$, qui augmenterait de façon significative avec un radôme) qui permet de l'installer sur une infrastructure légère, comme une tour télécom (voir figure 1). L'absence de radôme n'empêche pas une bonne tenue au vent de l'aérien, avec une résistance du positionneur allant jusqu'à $180 \mathrm{~km} / \mathrm{h}$ avec mise en survie (position à l'horizontale de l'antenne) au-delà.

Ce radar utilise le traitement $\mathrm{ZPHI}{ }^{\circledR}$ (Testud et al., 2000), qui tire profit du mode double polarisation du radar afin de corriger l'atténuation des mesures en présence de précipitation (voir encadré). Ce traitement est doté d'un

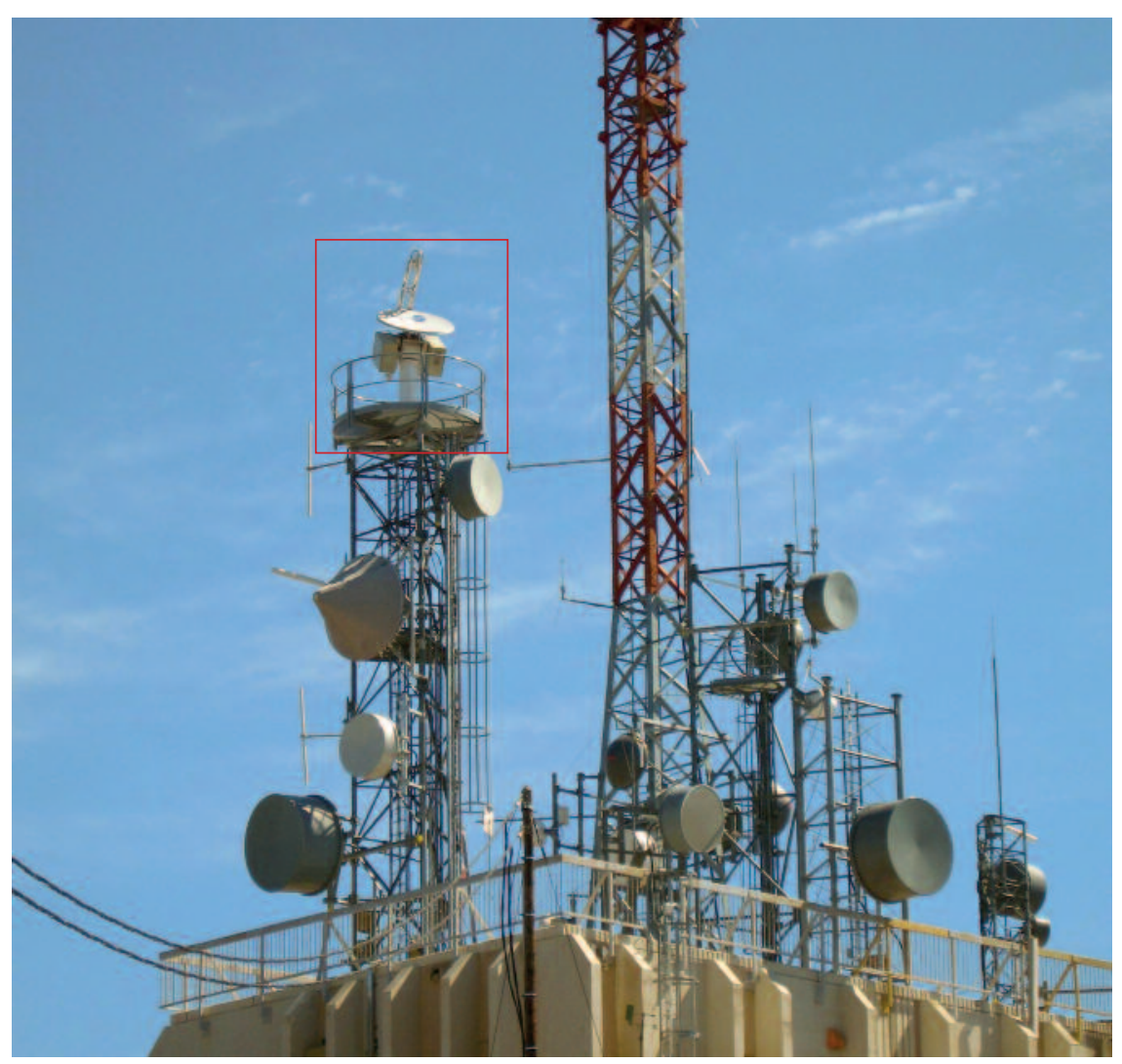

Figure 1. Implantation du radar Hydrix (cadre rouge) sur le mont Vial au nord de Nice. 
module permettant de séparer les échos de précipitations des échos parasites (échos de sol ou de mer) et de distinguer les différents types de précipitation (pluie, neige, neige fondante, grêle). L'algorithme ZPHI ${ }^{\circledR}$ permet également de corriger l'estimation de l'intensité de pluie des effets de variabilité naturelle de la pluie en adaptant automatiquement la relation de conversion de la réflectivité radar en intensité de pluie. Il permet, pour finir, une vérification de l'étalonnage absolu du radar en utilisant les mesures polarimétriques radar.

\section{La zone d'étude et les données}

La base de données exploitée dans ce travail couvre la période allant du début 2009 à la fin 2012. Ce radar fournit les mesures radar en coordonnées polaires toutes les 5 minutes et des produits dans un maillage cartésien avec une résolution spatiale de $1 \mathrm{~km}^{2}$, tels que la vitesse radiale, la réflectivité, le taux de pluie au sol instantané ou cumulé (sur $5 \mathrm{~min}, 1 \mathrm{~h}$ ou 24 h), le déplacement des cellules de précipitation, les paramètres polarimétriques et la classification des hydrométéores. La portée maximale est de 150 $\mathrm{km}$ autour du radar et la portée optimale de $60-80 \mathrm{~km}$.

La région d'étude est illustrée sur la figure 2. Elle regroupe cinq régions françaises et italiennes : Provence-AlpesCôte d'Azur, Ligurie, Piémont, Vallée d'Aoste et Rhône-Alpes.
Cette région est caractérisée par une forte variabilité des précipitations du fait de sa localisation géographique particulière qui la soumet aux effets de la ceinture de basses pressions des latitudes moyennes, en partie contrôlées par les oscillations de grande échelle comme l'oscillation nord-atlantique, et les anticyclones subtropicaux. En hiver, cette région est arrosée par les différents types de précipitations frontales des perturbations de moyenne latitude (Lemaître, 1987), tandis qu'en été l'anticyclone des Açores peut conduire à des périodes très sèches avec des événements de convection profonde dans l'après-midi. Cette région est également fortement affectée par des développements cyclogénétiques sur la mer. Elle est caractérisée par une forte hétérogénéité de la surface (mer, plaine, reliefs plus ou moins escarpés) et donc par des forçages de basse couche très variables (zones préférentielles de convergence de basse couche, de flux de chaleur latente et sensible, etc.).

Tous les processus dynamiques (thermodynamiques) sous-jacents à ces différents phénomènes vont gouverner la variabilité spatio-temporelle des précipitations associées. Cette variabilité spatiotemporelle s'effectue donc sur une grande gamme d'échelle et peut présenter une ampleur extrême (non gaussienne, non linéaire et chaotique).

La question qui se pose est donc de savoir s'il existe des processus qui permettraient d'expliquer des comportements systématiques, compte-tenu de la forte variabilité attendue des précipitations de cette région.

\section{Propriétés spatio-temporelles de la pluie}

\section{Propriétés statistiques}

Bien que les précipitations impliquent un large spectre de processus et présentent donc une grande complexité, plusieurs auteurs (Verrier et al., 2011 ; Lovejoy et al., 2012 ; Gires et al., 2012a ; Rysman et al., 2013, pour les travaux les plus récents) ont pu montrer de façon empirique que la variabilité des précipitations est caractérisée par une loi d'invariance d'échelle. Cette propriété se traduit par un spectre en puissance du taux précipitant $R$ qui suit une loi en puissance du type $P(f) \sim f^{-\beta}$ ou $T^{\beta}$ (avec $f$ la fréquence et $T$ la période) dont l'exposant $\beta$ reste constant sur une certaine gamme d'échelles. De telles symétries d'échelle sont assez analogues aux lois d'échelle classiques vérifiées par les champs turbulents (par ex. Kolmogorov, 1991). Cependant, dans le cas de la pluie, ce type de loi n'est valable que par intervalle de fréquence, notamment en raison de transitions météorologiques et de l'alternance entre périodes de pluie et périodes sèches (Fraedrich et Lardner, 1993 ; Fabry, 1996 ; Verrier et al., 2011 ; Rysman et al., 2013).

Ce comportement peut être analysé dans le cas présent sur le jeu de données Hydrix pour la période considérée à l'aide d'une analyse spectrale. La densité spectrale de puissance est obtenue par
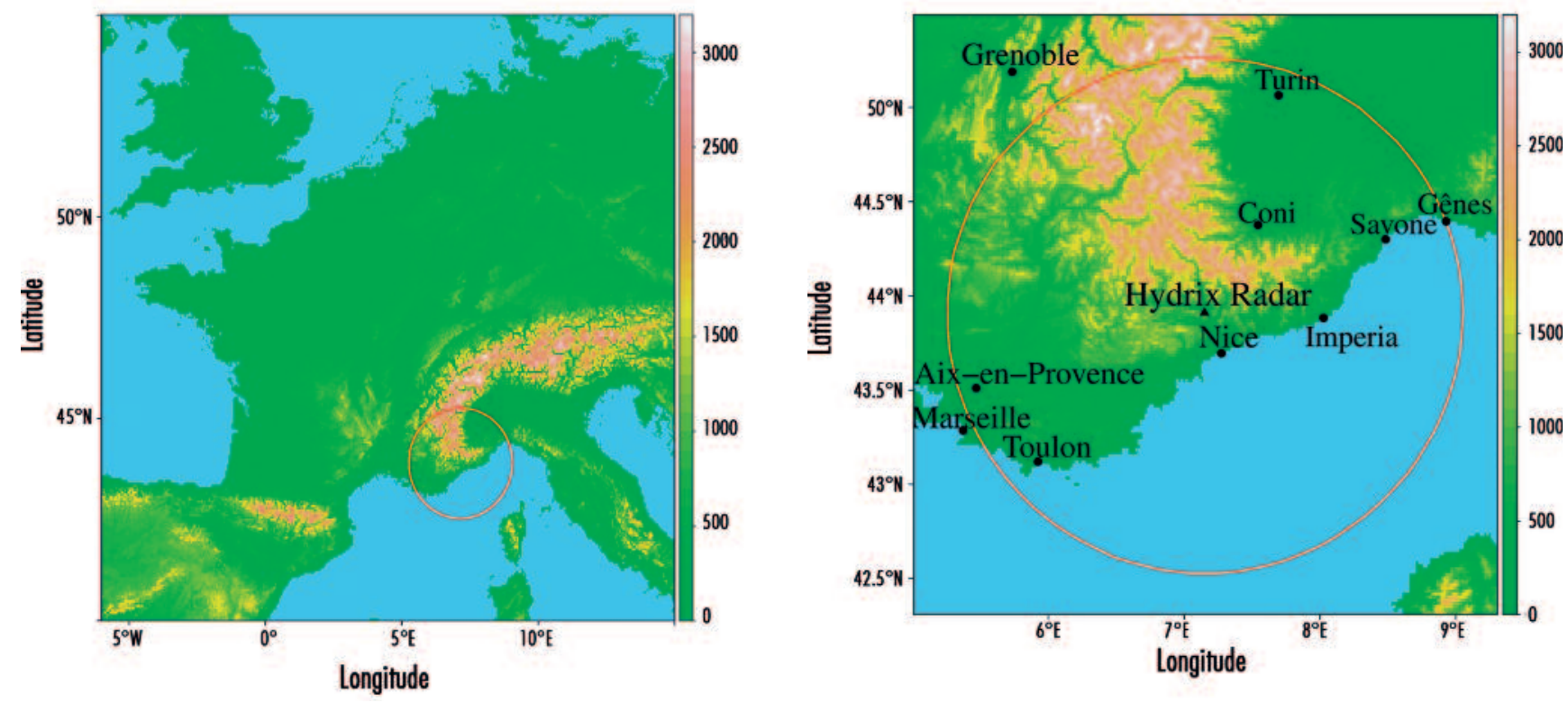

Figure 2. Localisation du radar Hydrix et couverture maximale (cercle rouge). L'échelle de droite, en couleurs, indique l'altitude. 
analyse de Fourier (dans le domaine temporel) du taux de pluie moyenné sur $5 \mathrm{~km}^{2}$ obtenu toutes les 5 minutes dans tout le champ de couverture radar et sur les quatre années d'observations. Le spectre temporel obtenu est donné en $\log$-log de la puissance et de la période (figure 3). On constate que le spectre (caractère d'un objet dans lequel on peut trouver des similarités en l'observant à différentes échelles) jusqu'à 10 jours et que plusieurs domaines d'invariance d'échelle (variation linéaire avec pente $\beta_{t}$ constante) peuvent être identifiés, typiquement entre 20 et $45 \mathrm{~min}$, entre $45 \mathrm{~min}$ et $3 \mathrm{~h}$ et entre 1 et dix jours. Les exposants de la loi en puissance obtenus par régression linéaire dans chacune de ces gammes d'échelles décroissent progressivement vers les grandes échelles de 1,31 à 0,46 en passant par 1,02 et 0,79 respectivement.

Une analyse équivalente à l'analyse temporelle précédente peut être réalisée dans le domaine spatial. La gamme d'échelles accessibles sera limitée dans ce cas par la couverture spatiale maximale du radar. La figure 4 donne le spectre spatial en $\log -\log$ de la puissance et de la longueur présente ce comportement autosimilaire

d'onde. Comme précédemment, on constate un caractère autosimilaire du spectre avec plusieurs domaines d'invariance d'échelle (variation linéaire avec pente $\beta_{x}$ constante) entre 3 et $7 \mathrm{~km}$, entre 7 et $20 \mathrm{~km}$ et entre 20 et plus de $70 \mathrm{~km}$. Les exposants de la loi de puissance obtenue par régression linéaire dans chacune de ces gammes d'échelles décroissent progressivement de 2 à 1,16 en passant par 1,5. En-dessous de $3 \mathrm{~km}$, la diminution de la pente résulte de l'effet du bruit de mesure et du filtrage par la grille radar (théorème d'échantillonnage de Nyquist-Shannon).

Ces différentes gammes d'échelles apparaissent proches de celles obtenues à partir d'une analyse en échelle de l'équation de la dynamique qui permet de faire apparaître des échelles caractéristiques dynamiques : convective $(\sim 10 \mathrm{~km}, \sim 30 \mathrm{~min})$, de mesoéchelle ( $\sim 100 \mathrm{~km}, \sim 3 \mathrm{~h})$, synoptique $(\sim 1000 \mathrm{~km}$, 1 jour) selon l'importance relative du terme d'accélération par rapport à la force de Coriolis. Cette analogie suggère que les processus présents à ces échelles gouvernent le comportement spectral de la pluie dans chacune de ces gammes d'échelles.

Figure 3. Densité spectrale d'énergie (taux de pluie) en fonction de la période (temporelle) en log-log. Le spectre est surmonté de lignes (noires) de pentes typiques déduites d'une régression linéaire.
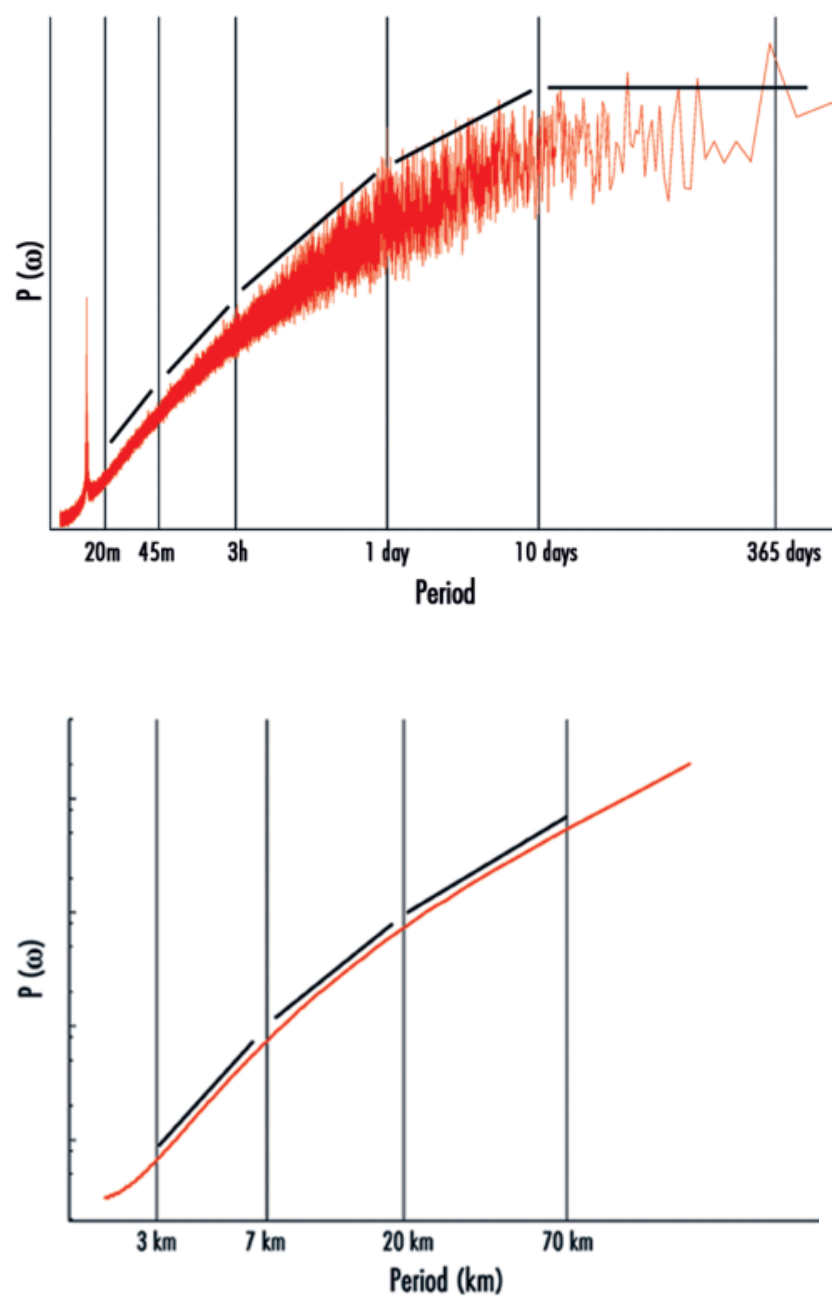

Fure 4. Densité spectrale d'énergie (taux de pluie) en fonction de la période (temporelle) en log-log. Le spectre est surmonté de lignes déduites d'une régression linéaire. (noires) de pentes typiques
Aux échelles inférieures à $10 \mathrm{~km}$ (30 min), le comportement spectral reflète principalement la variabilité associée à des événements continus de précipitation.

Aux fréquences spatiales et temporelles plus basses, la pente $\beta$ diminue progressivement. Pour ces gammes d'échelles, la pluie devient de plus en plus intermittente et le signal mesuré est dans ce cas la superposition d'un signal pluie et d'un processus d'occurrence (ou « support ») caractérisant cette intermittence pluie/ non-pluie. L'analyse spectrale donne donc dans ce cas les propriétés statistiques non seulement de la pluie (comme pour les échelles plus fines), mais également celles du support. En absence de support (c'est-à-dire d'intermittence pluie/non-pluie) et en présence de pluie continue à toute échelle, la pente spectrale serait constante et égale à celle obtenue aux petites échelles (au sein d'un même événement). Les changements de pentes sont donc associés au support qui apparaît ainsi fortement contrôlé par les échelles de mouvements et processus sous-jacents. Ce contrôle sur la structure des intermittences (spatiales ou temporelles) ne semble pas contradictoire avec le fait que les processus d'instabilité conduisent à des structures dynamiques (ascendance/ subsidence) qui ont des échelles caractéristiques définies par les processus (onde de gravité, onde gravito-inertielle, convection droite ou inclinée...) qui de ce fait déterminent les zones et périodes de précipitation et d'absence de précipitation.

Pour les gammes d'échelles supérieure à 10 jours, la pente du spectre est quasiment nulle (« plateau spectral », Fraedrich et Lardner, 1993). Ce plateau spectral de la mesure de précipitations à basse fréquence indique donc qu'audelà de 10 jours, il n'existe plus de corrélation temporelle entre deux événements et donc de loi permettant de relier ces échelles (ce qui correspond aussi à la limite possible de la prévision météorologique).

La justification théorique de ces lois d'échelle pour la pluie reste encore un sujet très ouvert (Lovejoy et Schertzer, 2008). Néanmoins, l'existence de lois de puissance et leur lien avec les échelles de mouvement et processus sous-jacents ouvrent des champs d'applications très intéressants déjà indiqués dans l'introduction. Ce lien devra cependant être étayé par des analyses réalisées sur des domaines spatiotemporels plus grands. L'acquisition continue de données du 
radar Hydrix dans les années qui viennent permettra d'étendre cette analyse sur une période décennale. Par contre, l'extension spatiale ne pourra se faire qu'en exploitant un réseau de radars. Une telle étude exploitant l'ensemble des données du réseau opérationnel est en cours et concerne l'ensemble du territoire français. Ces données permettront également d'étudier l'existence ou non d'une variabilité régionale de ces propriétés statistiques de la pluie.

Les lois d'échelle identifiées pour le spectre permettent de caractériser de façon statistique les transferts d'énergie entre échelles, ces transferts ayant lieu entre échelles voisines et de façon autosimilaire (Tessier et al., 1993). Ces relations entre échelles sont d'un grand intérêt pour réaliser de la descente d'échelle et permettent de relier des variables mesurées à petite échelle à des grandeurs mesurées à plus grande échelle (Rebora et al., 2006 ; Gires et al., $2012 b$ ). Les résultats présentés montrent cependant que cette descente d'échelle ne peut se réaliser qu'entre échelles (spatiales ou temporelles) contenues dans des gammes d'échelle présentant ce lien d'échelle : en-dessous de $7 \mathrm{~km}$ (20 min), entre 7 et $20 \mathrm{~km}$ (20-45 min), entre 20 et $70 \mathrm{~km}$ ( $45 \mathrm{~min}-3 \mathrm{~h}$ ) et entre 1 et 10 jours. La descente d'échelle pourra se réaliser entre échelles plus éloignées si l'on montre que les coupures entre gammes d'échelles sont fixes ou qu'il est possible de les déterminer a priori.

Les spectres d'énergie considérés cidessus offrent également une description fréquentielle de la pluie. Ces spectres permettent de mettre en évidence les caractéristiques de la variance du signal de pluie. Ils indiquent donc que cette variance dépend de la résolution spatiale et temporelle à laquelle on la calcule. D'un point de vue pratique, ceci montre que la comparaison entre deux mesures de pluie réalisées à des échelles spatiales

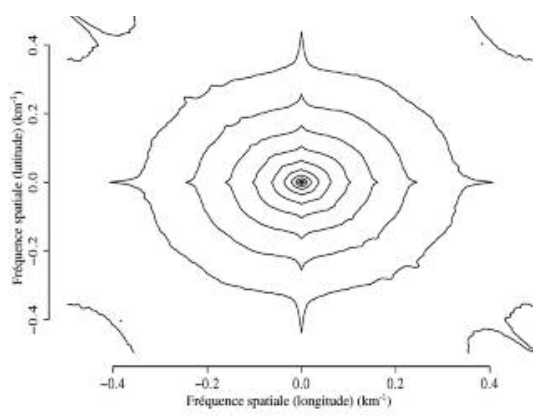

Figure 5. Courbe de niveau du logarithme de la densité spectrale de puissance (X-Y) pour la période juin 2009décembre 2011.

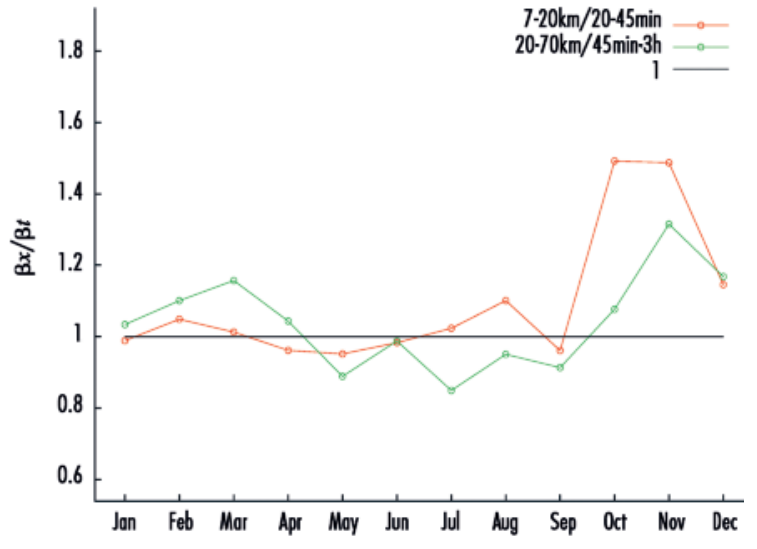

Figure 6. Évolution mensuelle du rapport des exposants spectraux $\beta_{x} / \beta_{t}$ pour la période début 2009-fin 2012 et pour les deux régimes 7-20 km (20-45 min) et $20-70 \mathrm{~km}$ (45 min-3 h).

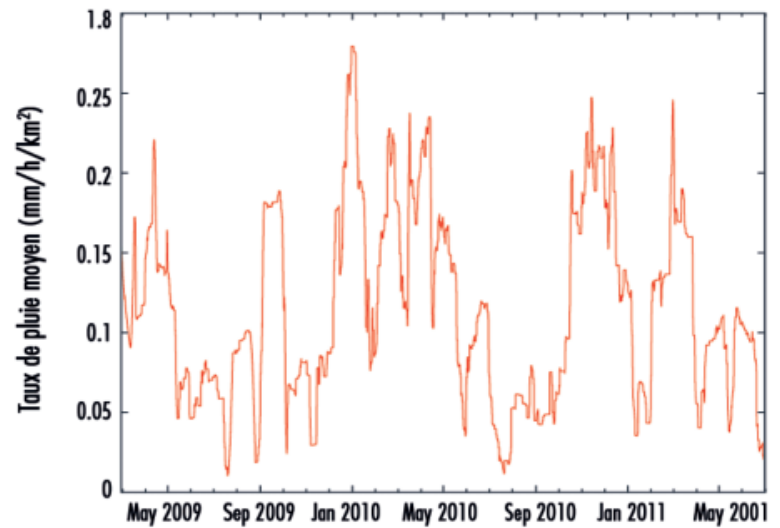

Figure 7. Évolution temporelle de la moyenne de cumul de pluie sur 5 minutes dans la zone de couverture radar entre février 2009 et juillet 2011.

et temporelles différentes à partir d'instruments de résolutions différentes, in situ, de télédétection au sol ou spatial, est difficile (problème de représentativité de la mesure) et ne peut se réaliser en toute connaissance de cause qu'en prenant en compte cette loi d'échelle (Verrier et al., 2013), avec les précautions indiquées précédemment.

Létude des données Hydrix révèle d'autres informations au sujet de l'équivalence ou de la non-équivalence des axes spatiaux et temporels considérés. On constate, comme le montre la figure 5 à l'aide d'une analyse spectrale bidimensionnelle du jeu de données, qu'aucune direction (spatiale) privilégiée n'apparaît (Rysman et al., 2013) et que donc ces propriétés d'échelle (lien entre échelle) sont invariantes par toute rotation autour d'un axe (isotropie).

Ce jeu de données permet également d'étudier l'anisotropie qui peut exister entre le lien entre échelles spatiales et le lien entre échelles temporelles. La connaissance de ces relations d'échelle spatio-temporelle permettrait de savoir si la connaissance des propriétés statistiques à haute résolution dans le temps (ou dans l'espace) pourrait être utilisée pour inférer les propriétés statistiques à haute résolution dans l'espace (ou le temps). On pourrait ainsi utiliser l'information à haute résolution dans le temps (ou l'espace) pour interpoler l'information à basse résolution dans l'espace (ou le temps).

Afin d'étudier cette anisotropie, le rapport $\beta_{x} / \beta_{t}$ entre pente spatiale et temporelle du spectre en puissance est calculé. $\mathrm{Ce}$ calcul s'effectue pour les deux régimes autosimilaires indiqués précédemment 7-20 km (20-45 min) et $20-70 \mathrm{~km}$ (45 min-3 h) et pour la période de quatre années considérée. Les résultats sont présentés en figure 6 . Le rapport proche de 1 obtenu pour la majeure partie de l'année (excepté en automne) pour les deux régimes indique qu'aucune anisotropie entre les liens entre échelles spatiales et les liens entre échelles temporelles n'est présente. La constance de ce rapport suggère que ces liens portent bien sur les mêmes processus météorologiques pour ces deux régimes. Les résultats obtenus en automne doivent être pris avec précaution en raison d'un possible sous-échantillonnage temporel des événements précipitants de cette période, du fait qu'ils sont très souvent localisés sur la 
mer et se déplacent rapidement et donc se retrouvent rapidement hors de la zone de couverture du radar.

\section{Variabilités spatiales et temporelles}

Comme indiqué en introduction, les précipitations méditerranéennes sont gouvernées par de nombreux processus dynamiques et thermodynamiques qui déterminent la variabilité spatiotemporelle des précipitations. Cette variabilité spatio-temporelle s'effectue sur une large gamme d'échelle. Le jeu de données acquis jusqu'à maintenant permet de couvrir des variabilités temporelles (et les processus sous-jacents) qui vont de quelques minutes à plusieurs années.

La figure 7 donne l'évolution temporelle de la moyenne de cumul de pluie sur 5 min dans la zone de couverture radar entre mars 2009 et juillet 2011. Elle montre l'existence de cycles avec pour chaque année en moyenne une décroissance du taux précipitant de janvier à juillet et avec un minimum en août sur laquelle se superpose un cycle de 1 à 2 mois plus marqué en 2010.

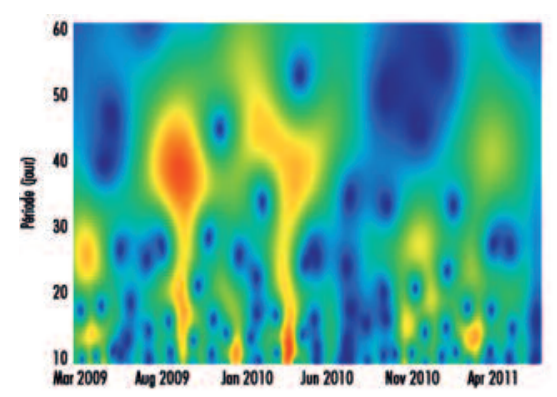

Figure 8. Analyse en ondelettes de la pluie cumulée sur 5 minutes entre mars 2009 et juillet 2011.

Les modes principaux de cette variabilité temporelle peuvent être identifiés plus précisément en utilisant une analyse en ondelette. Cette analyse permet une analyse en fréquence et en temps du signal (cumul de pluie sur 5 min dans la zone de couverture radar dans le cas présent). Elle permet ainsi la localisation de changements abrupts dans le comportement du signal et la détection de comportement oscillatoire. La figure 8 donne un exemple de résultat pour la période mars 2009- juillet 2011 et pour des ondelettes (de type Morlet) de périodes comprises entre 1 à 60 jours. Cette figure donne l'énergie de l'ondelette (le taux de pluie moyen) en fonction de la période (en ordonnée) et du jour (en abscisse).
Plusieurs modes apparaissent pendant cette période, en particulier un mode à 30-40 jours plus actifs en juin-octobre 2009 et janvier-avril 2010 et un mode à 6-25 jours (d'autres modes apparaissent à des périodes plus grandes, mode annuel, cycle à 80-130 jours, etc.). L'obser-vation de tels modes montre que la pluie dans la zone d'étude est sous l'influence de structure de plus grande échelle (de type oscillation nord-atlantique, oscillation de Madden-Julian...).

L'analyse de la variabilité spatiale de ces précipitations est également importante. Elle permet par exemple d'évaluer le rôle de l'orographie sur les lieux préférentiels d'activité pluvieuse intense (maximum de taux précipitant) ou d'impact hydrométrique (maximum de cumul de pluie) locaux. L'orographie peut modifier en effet localement les circulations de basse couche par canalisation, par soulèvement, etc. ou en altitude par excitation d'ondes de relief, de gravité, etc. et créer ainsi ces lieux privilégiés. Une façon d'analyser ce rôle local est de quantifier la probabilité de présence en un lieu géographique donné d'événements précipitants intenses (en terme de taux de pluie instantané ou de cumul de pluie). Pour ce faire, les séries de pluie (taux précipitant ou cumul) mesurée dans la zone de couverture du radar sont classifiées en utilisant un critère de dépassement d'un quantile choisi afin d'isoler la partie extrême de la distribution. Ainsi, le jeu d'événements pluvieux significatifs est constitué pour des valeurs de quantiles dépassant Q99,99 (quantile 99,99\%).

La figure 9 donne un exemple de répartition spatiale de ces événements significatifs en terme de pluie cumulée sur 5 minutes pour l'ensemble des quatre années d'observation du radar Hydrix (sans prendre donc en compte les variabilités temporelles annuelle, saisonnière, etc.). Elle montre un signal très marqué

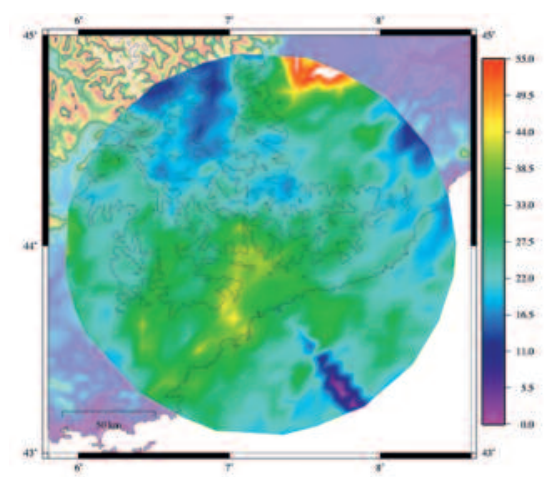

Figure 9. Répartition spatiale des événements de pluie significatifs (en terme de cumul sur 5 minutes) pour les quatre années d'observation du radar Hydrix. avec deux régions préférentielles situées dans la vallée d'Aoste en Italie (région en bout de portée radar pour laquelle la quantification précise des précipitations est difficile) et la région Provence-AlpesCôte d'Azur entre Sainte-Maxime et Menton. La première région, bien connue, résulte de la convergence d'air humide et chaud sur la plaine du Pô en provenance de la Méditerranée, qui est canalisé et concentré dans cette région par le relief montagneux. La deuxième région en Provence-Alpes-Côte d'Azur semble forcée par le bord sud du relief alpin et la transition terre-mer de cette région.

Dans la suite de cet article est donné deux exemples de tels cas de pluie intense documentés par le radar lors de la première campagne d'observation SOP1 (période d'observation spéciale 1) du programme HyMeX (http://www. hymex. org). Ce programme de recherche international vise à progresser dans les connaissances sur le cycle hydrologique du bassin méditerranéen avec pour objectif d'améliorer la prévision et la prévention des phénomènes intenses en Méditerranée (pluies intenses, crues rapides, vents forts...) et d'améliorer la prévision régionale des impacts du changement climatique.

\section{Exemples d'événements intenses observés par le radar Hydrix}

Ces événements sont deux exemples de précipitations observées en 2012 les 12 et 14 octobre dans la région ProvenceAlpes-Côte d'Azur. Ces deux exemples illustrent la qualité des produits disponibles dans la base de données Hydrix pour des études statistiques et des études de cas.

L'événement du 12 octobre correspond à la période d'observations intensives POI 12a de la SOP1 d'HyMeX (figure 10). Il résulte d'un talweg en altitude associé à une zone de convergence d'air chaud et humide dans les basses couches alimentée par un flux de sud qui initie et alimente des cellules orageuses et une succession de systèmes convectifs de mésoéchelle. Le premier de ces systèmes de mésoéchelle observé par Hydrix se présente à 00 h35 (heure légale) le 12 octobre 2012 comme une ligne de précipitation très étroite et intense, parallèle à la côte, dont les taux 
de précipitation dépassent les $140 \mathrm{~mm} / \mathrm{h}$ (figure 11). Il conduit à la formation de grêle (figure 12) comme l'indique l'analyse des mesures polarimétriques du radar. La détermination de ce contenu particulaire (hydrométéore) se réalise grâce à un algorithme d'identification ou de classification des hydrométéores, c'est-à-dire par l'association d'un type ou d'une classe d'hydrométéores à un ensemble de valeurs des variables polarimétriques via une approche de logique floue (Straka et al., 2000 ; Le Bouar et al., 2011). Ces mesures polarimétriques permettent en effet de quantifier différentes variables caractérisant les propriétés microphysiques des hydrométéores.
Parmi celles-ci, on dispose de la réflectivité différentielle ZDR, rapport exprimé en décibels sous la forme $\mathrm{ZDR}=10 \log \left(Z_{\mathrm{h}} / Z_{\mathrm{v}}\right)$ avec $Z_{\mathrm{h}}$ et $Z_{\mathrm{v}}$ respectivement les réflectivités radar en polarisation horizontale et verticale. Il informe sur la taille et la forme moyenne des hydrométéores. Il peut par exemple être utilisé pour distinguer l'état solide de l'état liquide. Pour des particules sphériques (gouttelettes, grêle) ou qui ne montrent pas pendant leur chute de direction préférentielle (neige, cristaux de glace), ZDR est proche de zéro. Dans le cas contraire, la forme oblate des gouttes de pluie provoque une diffusion anisotrope de l'onde incidente et ZDR positif devient d'autant plus grand que les gouttes sont grosses, donc aplaties. De même, le facteur de corrélation $\rho_{\mathrm{hv}}$ permet d'inférer statistiquement la variabilité microphysique de la cible. Plus la diversité en taille, forme, orientation, nature et type d'hydrométéores est importante, plus ce facteur est petit. Cette classification fait partie de la chaîne de traitement ZPHI ${ }^{\circledR}$ (voir encadré).

À ces paramètres polarimétriques s'ajoute la vitesse Doppler (figure 13) qui donne accès au champ de vent à l'aide de différentes méthodes d'inversion dont celles indiquées en introduction.

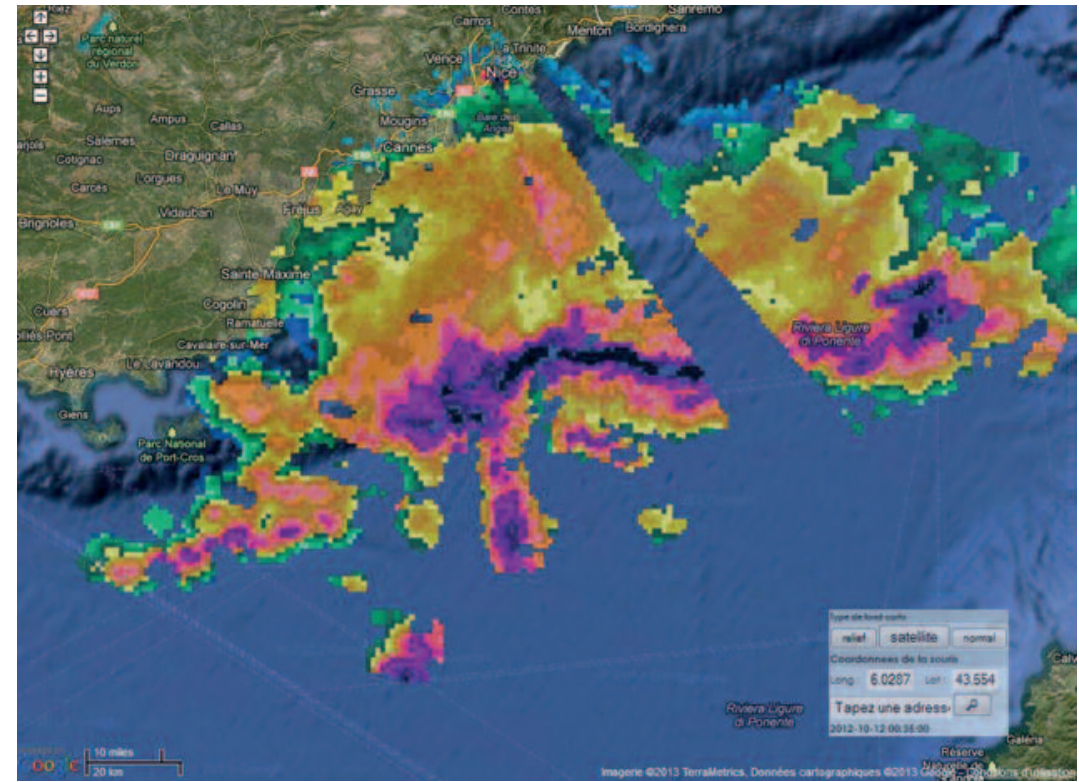

Figure 10. Carte de réflectivité radar corrigée de l'atténuation à $-1^{\circ}$ d'élévation le 12 octobre 2012 à 00 h35 (heure légale). Le secteur sud-est sans réflectivité résulte du masque induit par la tour de télécommunication contigüe au radar (voir figure 2). Les zones en noire correspondent à des réflectivités supérieures à $56 \mathrm{dBZ}$

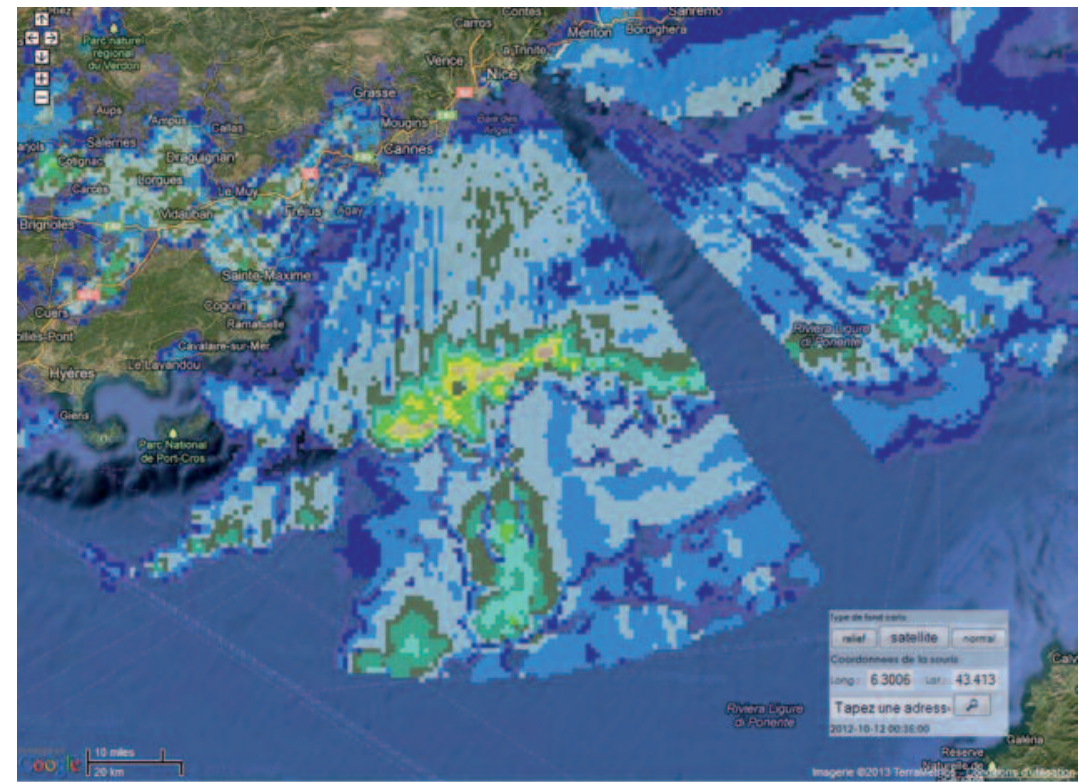

Figure 11. Carte de cumul de pluie sur 5 minutes au sol le 12 octobre 2012 à 00 h35 (heure légale). Les zones en beige correspondent à des cumul de l'ordre de $10 \mathrm{~mm}$.

\section{Traitement de la lame d'eau radar ZPHI@}

La chaîne de traitement ZPHI® suit plusieurs étapes :

1. Qualification des mesures polarimétriques et correction des biais éventuels dus à l'étalonnage du radar et aux masques orographiques.

2. Classification : avec une première étape d'élimination des échos non météorologiques, puis une deuxième étape de classification en cinq catégories (pluie stratiforme, pluie convective, neige fondante, neige sèche et grêle) par une approche de logique floue. Les fonctions d'appartenance sont obtenues à partir de modèles de diffusions (Mie, Aden-Kerker, TMatrix) pour les différents types d'hydrométéores (voir Le Bouar et al. (2011) sur la classification de la grêle).

3. Correction d'atténuation : la réflectivité absolue $Z$ et la réflectivité différentielle ZDR sont corrigées de l'atténuation par la pluie au moyen de I'algorithme ZPHI®.

4. Estimation du taux de pluie : la variabilité naturelle de la distribution des particules de pluie est prise en compte dans l'estimation du taux de pluie à travers un paramètre. Ce paramètre est estimé par I'algorithme ZPHI® de façon systématique et pour chaque type de pluie.

5. Lame d'eau au sol : en chaque point de grille de la carte de pluie, l'intensité de pluie au sol est calculée comme la somme pondérée des intensités de pluie estimées aux différentes altitudes (cinq élévations entre $-1^{\circ}$ et $+5^{\circ}$ ). La pondération est fonction de l'altitude de la mesure par rapport au sol, du masque orographique présent, de l'échantillonnage temporel et de l'algorithme d'estimation du taux de pluie utilisé. 
L'événement du 14 octobre 2012 (POI 13 d'HyMeX) s'est développé dans le flux de basse couche chaud et humide de sudouest situé dans le secteur chaud d'une perturbation frontale.

Il correspond également à une situation de pluies intenses dont le système orageux le plus à l'ouest de la zone de couverture radar a généré vers $16 \mathrm{~h}$ une tornade qui a balayé pendant une dizaine de minutes la zone de Plan-de-Campagne, près de Marseille, faisant une vingtaine de blessés légers et occasionnant de nombreux dégâts matériels. Bien qu'issue de structures nuageuses préfrontales orientées sudouest à nord, la région de précipitation intense apparaît fortement contrôlée par la transition terre-mer et le relief (figure 14).

\section{Conclusions et perspectives}

L'avènement de mesures par radar Doppler et polarimétrique en bande $\mathrm{X}$ dans des régions montagneuses à fort contraste orographique (actuellement faiblement couvertes par les réseaux de mesures de pluie au sol ou de façon incomplète par le réseau de radars conventionnel) dont la topographie contrôle fortement la variabilité des précipitations est d'un très grand intérêt. Les études réalisées dans le passé ont permis de confirmer l'intérêt des radars en bande X pour la connaissance spatialisée des pluies. Elles ont également montré l'intérêt de la double polarisation pour la mesure quantitative de la pluie. Le projet de Météo-France de se doter progressivement de radars de ce type (Westrelin et al., 2012) pour la prévision des aléas naturels liés aux précipitations permettra, pour des applications de recherche, d'améliorer nos connaissances sur la dynamique et la microphysique des systèmes pluvieux orographiques. Le potentiel d'application dans ce domaine, comme nous espérons l'avoir montré, est important. Il nécessite encore cependant de progresser sur l'exploitation de ces mesures en particulier la mesure Doppler. Pour ce qui concerne le radar Hydrix, sa mise en œuvre de façon continue, dans le cadre de la coopération CNRS/ Novimet en cours, sur des périodes suffisamment longues (décennales) permettra de se pencher sur les tendances climatiques à moyenne échéance en terme de précipitations de la région Alpes-Méditerranée. Il $\mathrm{y}$ a en effet un besoin de suivi à long terme pour identifier et quantifier ces tendances et une demande sociétale forte en vue de l'adaptation nécessaire au changement climatique déjà en cours.

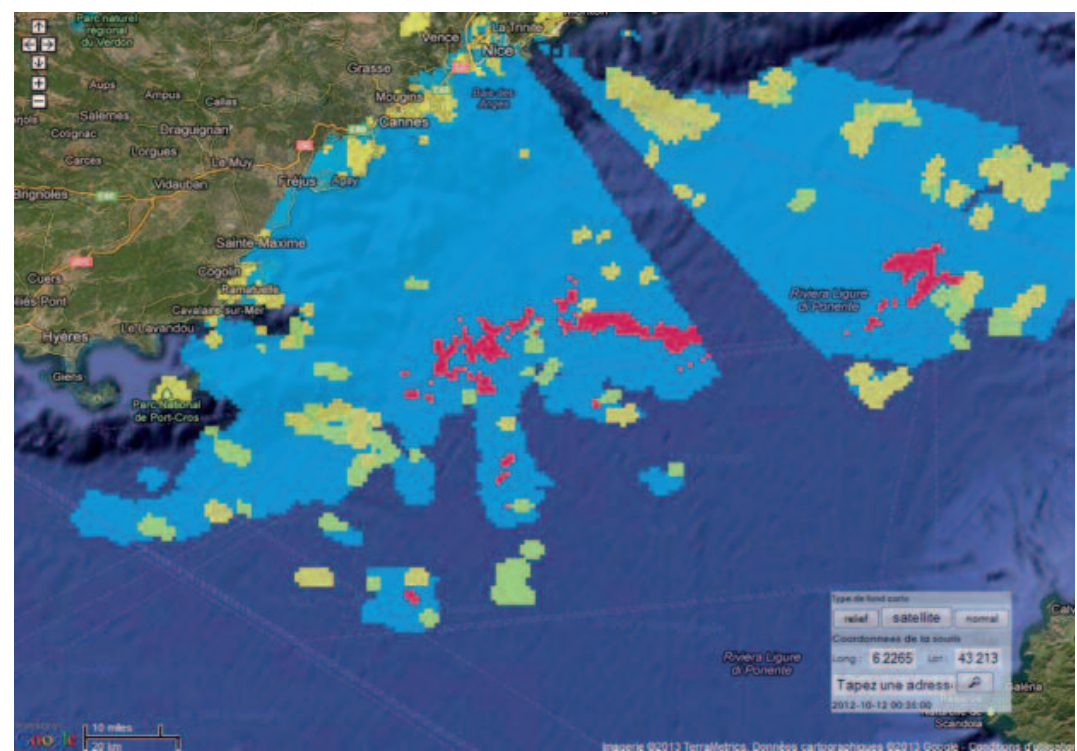

Figure 12. Carte de classification des hydrométéores à $-1^{\circ}$ d'élévation le 12 octobre 2012 à 00 h35 (heure légale). Les zones en rouge indiquent la présence de grêle, en bleu de pluie et en jaune d'échos de mer/sol.

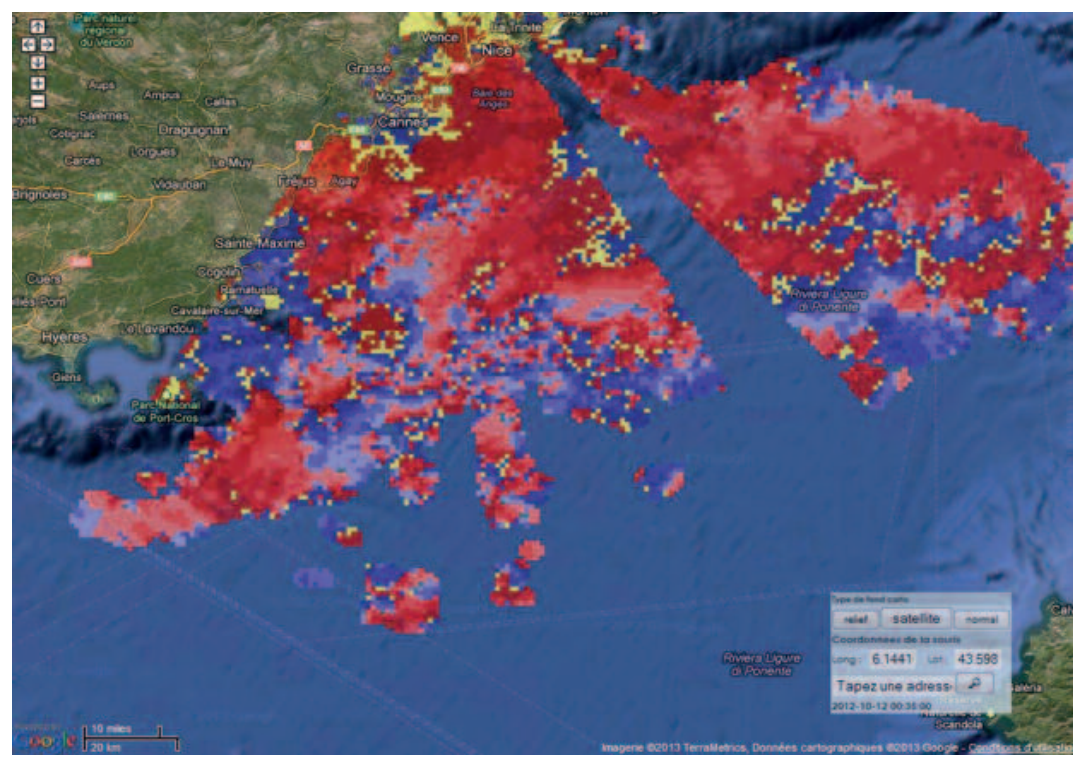

Figure 13. Carte de vitesse Doppler non dépliée à $-1^{\circ}$ d'élévation le 12 octobre 2012 à 00 h35 (heure légale). Le domaine de vitesse non ambiguë va de -5 à $5 \mathrm{~ms}^{-1}$. Les zones en rouge (jaune) correspondent à des valeurs de l'ordre de $3 \mathrm{~ms}^{-1}\left(0 \mathrm{~ms}^{-1}\right)$.

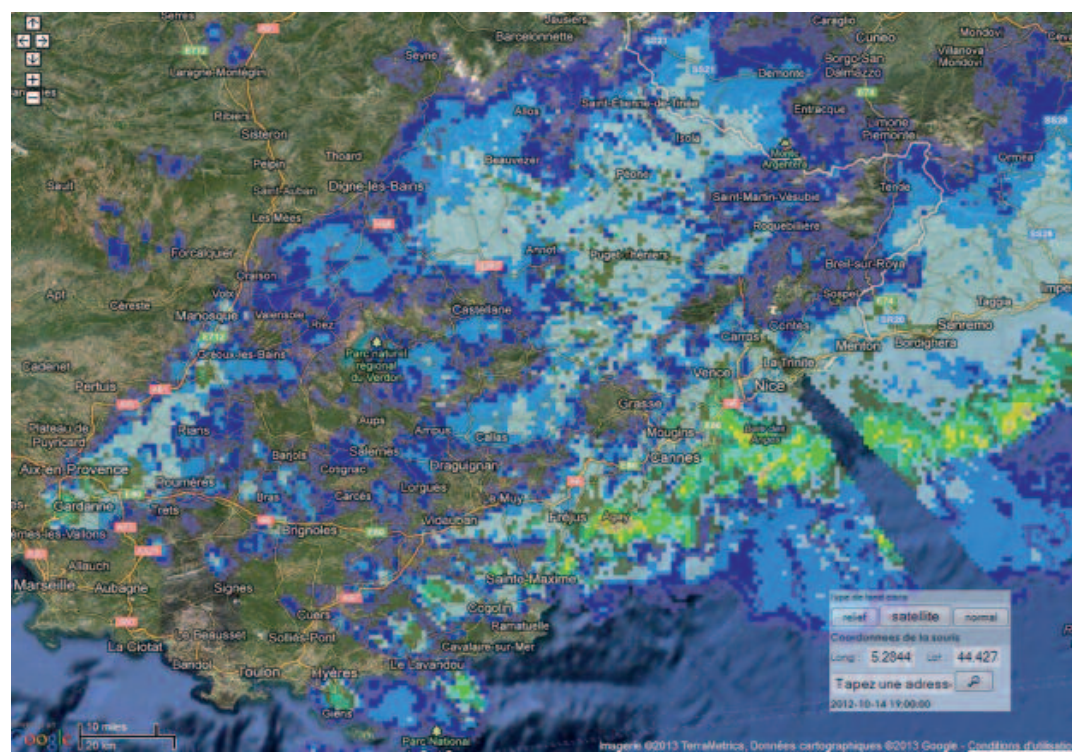

Figure 14. Carte de cumul de pluie sur 5 minutes au sol le 14 octobre 2012 à 19 h (heure légale). La cellule orageuse située à l'ouest a donné naissance à une tornade sur Marseille trois heures plus tôt. Les zones en beige correspondent à des cumul de l'ordre de $10 \mathrm{~mm}$. Les zones en beige correspondent à des cumul de l'ordre de $10 \mathrm{~mm}$. 


\section{Bibliographie}

Beck J. et 0. Bousquet, 2013 : Using gap-filling radars in mountainous regions to complement a national radar network: improvements in multiple-doppler wind syntheses. J. Appl. Meteorol. Clim., 52, 1836-1850.

Chong M. et J. Testud, 1996 : Three-dimensional air circulation in a squall line from airborne dual-beam Doppler radar data: a test of coplane methodology software. J. Atmos. Oceanic Technol., 13, 36-53.

de Montera L., L. Barthès, C. Mallet et P. Golé, 2009 : The effect of rain-no rain intermittency on the estimation of the universal multifractals model parameters. J. Hydrometeorol., 10, 493506.

Diss S., J. Testud, J. Lavabre, P. Ribstein, E. Moreau et J. Parent du Chatelet, 2009 : Ability of a dual polarized X-band radar to estimate rainfall. Adv. Water Resour., 32, 975-985.

Drobinski P., V. Ducrocq, P. Arbogast, C. Basdevant, S. Bastin, L. Beguery, S. Belamari, K. Béranger, O. Bock, O., M.-N. Bouin, B. Boudevillain, 0. Bousquet, C. Bouvier, I. Braud, J.-C. Calvet, C. Champollion, A. Chanzy, M. Claude, P. Cocquerez, L. Coppola, S. Coquillat, D. Courault, E. Defer, J. Delanoë, G. Delrieu, A. Doerenbecher, A. Dorfliger, M. Estèves, C. Estournel, C. Flamant, N. Fourrié, O. Garrouste, E. Gaume, H. Giordani, L. Gomes, G. Roberts, H. Jourde, L. Labatut, D. Lambert, J. Le Coz, W. Ludwig, C. Lutoff, C., J.-F. Mahfouf, E. Martin, L. Mastrorillo, N. Mathys, Y. Michel, M. Nuret, F. Orain, S. Perez, C. Prigent, O. Radakovitch, K. Ramage, E. Richard, J.-L. Roujeau, O. Roussot, I. Ruin, F. Saïd, A. Schwarzenböck, K. Sellegri, S. Somot, I. Taupier-Letage, P. Tes-tor, J.S. Van Baelen et J.-P. Vandervaere, 2013 : HyMeX, le cycle de l'eau méditerranéen à la loupe. La Météorologie, 80, 23-36.

Ducrocq V., S. Belamari, B. Boudevillain, O. Bousquet, P. Cocquerez, A. Doerenbecher, P. Drobinski, C. Flamant, L. Labatut, D. Lambert, M. Nuret, E. Richard, O. Roussot, P. Testor, P. Arbogast, P.A. Ayral, J. S. Van Baelen, C. Basdevant, J.-L. Boichard, D. Bourras, C. Bouvier, M.-N. Bouin, O. Bock, I. Braud, C. Champollion, L. Coppola, S. Coquillat, E. Defer, J. Delanoë, G. Delrieu, J.-F. Didon-Lescot, P. Durand, C. Estournel, N. Fourrié, O. Garrouste, H. Giordani, J. Le Coz, Y. Michel, O. Nuissier, G. Roberts, F. Said, A. Schwarzenböck, K. Sellegri, I. Taupier-Letage et J.-P. Vandervaere, 2013 : HyMeX, les campagnes de mesures : focus sur les événements extrêmes en Méditerranée. La Météorologie, 80, 37-47.

Fabry F., 1996 : On the determination of scale ranges for precipitation fields. J. Geophys. Res., 101, 12819-12826.

Fraedrich K. et C. Larnder, 1993 : Scaling regimes of composite rainfall time series. Tellus, 45, 289-298.

Frasier S., F. Kabeche, J. Figueras i Ventura, H. Al-Sakka, P. Tabary, J. Beck et O. Bousquet, 2013 : In-situ estimation of wet-radome attenuation at X-band. J. Atmos. Ocean. Technol., 30, 917-928.

Gires A., I. Tchiguirinskaia, D. Schertzer et S. Lovejoy, 2012a : Influence of the zero-rainfall on the assessment of the multifractal parameters. Adv. Wat. Resour., 45, 13-25.

Gires A., C. Onof, C. Maksimovic, D. Schertzer, I. Tchiguirinskaia et N. Simoes, 2012b : Quantifying the impact of small scale unmeasured rainfall variability on urban runoff through multifractal downscaling: A case study. J. Hydrol., 442-443, 117-128.

Kolmogorov A.N., 1991 : Local structure of turbulence in incompressible viscous fluid for very large Reynolds numbers. Proc. R. Soc. London, A, 434, 9-13 (traduit de l'original publié en 1941 dans Proc. Acad. Sci. URSS, 30(4)).

IPCC, 2007 : Summary for Policymakers. In: Climate Change, 2007: The Physical Science Basis. Contribution of Working Group I to the Fourth Assessment Report of the Intergovernmental Panel on Climate Change [Solomon S., D. Qin, M. Manning, Z. Chen, M. Marquis, K.B. Averyt, M.Tignor et H.L. Miller (eds.)]. Cambridge University Press, Cambridge, United Kingdom and New York, NY, USA. $18 \mathrm{p}$.

Le Bouar E., E. Moreau et J. Testud, 2011 : Hail events observed by an X-band polarimetric radar along the French Mediterranean coast. Weath. Radar Hydrol., IAHS Publ. 351, 99-104. Lemaître Y., 1987 : Phénomènes de moyenne échelle dans les systèmes frontaux, La Météorologie, VII série, 18, 25-37.

Lovejoy S. et D. Schertzer, 2008 : Turbulence, rain drops and the 11/2 number density law. New J. Phys., 10, 075017.

Lovejoy S., J. Pinel et D. Schertzer, 2012 : The global space-time cascade structure of precipitation: satellites, gridded gauges and reanalyses. Adv. Water Resour., 45, 37-50.

Michelangeli P.-A., M. Vrac et H. Loukos, 2009 : Probabilistic downscaling approaches: Application to wind cumulative distribution functions. Geophys. Res. Lett., 36, L11078, doi: 10.1029/2009GL028401.

Moreau E., J. Testud et E. Le Bouar, 2009 : Rainfall spatial variability observed by X-band weather radar and its implication for the accuracy of rainfall estimates. Adv. Water Resour., 32, 1011-1019.

Rebora N., L. Ferraris, J. Von Hardenberg et A. Provenzale, 2006 : Rainfall downscaling and flood forecasting: a case study in the Mediterranean area. Nat. Haz. Earth Syst. Sci., 6, 611-619. Rysman J.-F., S. Verrier, Y. Lemaître et E. Moreau, 2013 : Space-time variability of the rainfall over the western Mediterranean region: A statistical analysis. J. Geophys. Res. Atmos., 118, 8448-8459.

Scialom G. et Y. Lemaître, 1990 : A new analysis for the retrieval of three-dimensional mesoscale wind fields from multiple Doppler radar. J. Atmos. Oceanic Technol., 7, 640-665.

Straka J.M., D.S. Zrni et A.V. Ryzhkov, 2000 : Bulk hydrometeor classification and quantification using polarimetric radar data: synthesis of relation. J. Appl. Meteor., 39, 1341 -1372.

Tessier Y., S. Lovejoy et D. Schertzer, 1993 : Universal multifractals theory and observations for rain and clouds. J. Appl. Meteorol., 32, $223-250$.

Testud J., E. Le Bouar, E. Obligis et M. Ali-Mehenni, 2000 : The rain profiling algorithm applied to polarimetric weather radar. J. Atmos. Ocean. Technol., 17, 332-356.

Verrier S., C. Mallet et L. Barthès, 2011 : Multiscaling properties of rain in the time domain, taking into account rain support biases, J. Geophys. Res., 116, D20119, doi:10.1029/2011JD015719.

Verrier S., L. Barthès et C. Mallet, 2013 : Theoretical and empirical scale dependency of Z-R relationships: Evidence, impacts, and correction, J. Geophys. Res Atmos.., 118, 7435-7449. Vrac M., P. Drobinski, A. Merlo, M. Herrmann, C. Lavaysse, L. Li et S. Somot, 2012 : Dynamical and statistical downscaling of the French Mediterranean climate: uncertainty assessment. Nat. Haz. Earth Syst. Sci., 12, 2769-2784.

Westrelin S., P. Mériaux, P. Tabary et Y. Aubert, 2012 : RHYTMME Project: Risk management based on a radar network. Proceedings ERAD 2012, Toulouse, France. 